\title{
On the cytokine/chemokine network during Plasmodium vivax malaria: new insights to understand the disease
}

\author{
Natália Satchiko Hojo-Souza ${ }^{1 \dagger}$, Dhelio Batista Pereira ${ }^{2 \dagger}$, Fernanda Sumika Hojo de Souza ${ }^{3}$, \\ Tiago Antônio de Oliveira Mendes ${ }^{4}$, Mariana Santos Cardoso ${ }^{1}$, Mauro Shugiro Tada ${ }^{2}$, Graziela Maria Zanini ${ }^{5}$, \\ Daniella Castanheira Bartholomeu', Ricardo Toshio Fujiwara' and Lilian Lacerda Bueno ${ }^{{ }^{*}}$
}

\begin{abstract}
Background: The clinical outcome of malaria depends on the delicate balance between pro-inflammatory and immunomodulatory cytokine responses triggered during infection. Despite the numerous reports on characterization of plasma levels of cytokines/chemokines, there is no consensus on the profile of these mediators during blood stage malaria. The identification of acute phase biomarkers might contribute to a better understanding of the disease, allowing the use of more effective therapeutic approaches to prevent the progression towards severe disease. In the present study, the plasma levels of cytokines and chemokines and their association with parasitaemia and number of previous malaria episodes were evaluated in Plasmodium vivax-infected patients during acute and convalescence phase, as well as in healthy donors.
\end{abstract}

Methods: Samples of plasma were obtained from peripheral blood samples from four different groups: P. vivaxinfected, P. vivax-treated, endemic control and malaria-naïve control. The cytokine (IL-6, IL-10, IL-17, IL-27, TGF- $\beta$, IFN- $\gamma$ and TNF) and chemokine (MCP-1/CCL2, IP-10/CXCL10 and RANTES/CCL5) plasma levels were measured by CBA or ELISA. The network analysis was performed using Spearman correlation coefficient.

Results: Plasmodium vivax infection induced a pro-inflammatory response driven by IL-6 and IL-17 associated with an immunomodulatory profile mediated by IL-10 and TGF- $\beta$. In addition, a reduction was observed of IFN- $\gamma$ plasma levels in P. vivax group. A lower level of IL-27 was observed in endemic control group in comparison to malaria-naïve control group. No significant results were found for IL-12p40 and TNF. It was also observed that P. vivax infection promoted higher levels of MCP-1/CCL2 and IP-10/CXCL10 and lower levels of RANTES/CCL5. The plasma level of IL-10 was elevated in patients with high parasitaemia and with more than five previous malaria episodes. Furthermore, association profile between cytokine and chemokine levels were observed by correlation network analysis indicating signature patterns associated with different parasitaemia levels.

Conclusions: The P. vivax infection triggers a balanced immune response mediated by IL-6 and MCP-1/CCL2, which is modulated by IL-10. In addition, the results indicated that IL-10 plasma levels are influenced by parasitaemia and number of previous malaria episodes.

Keywords: Plasmodium vivax, Malaria, Cytokines, Chemokines

\footnotetext{
*Correspondence: Ilbueno@icb.ufmg.br

${ }^{\dagger}$ Natália Satchiko Hojo-Souza and Dhelio Batista Pereira contributed equally to this work

${ }^{1}$ Departamento de Parasitologia, Instituto de Ciências Biológicas,

Universidade Federal de Minas Gerais, Belo Horizonte, Minas Gerais, Brazil

Full list of author information is available at the end of the article
} 


\section{Background}

Malaria is caused by a protozoan of the genus Plasmodium and is responsible for high morbidity rates (besides the cases of mortality, especially among children), resulting in serious impact on the socio-economic development in endemic regions. In the Brazilian Amazon region, Plasmodium vivax is the main species causing malaria, being responsible for $82 \%$ of the cases [1]. Malaria is a complex disease involving genetic factors inherent to the parasite and to the host, geographical and environmental aspects that favour its occurrence and the difficulty of its eradication [2].

During infection, both the antibody-mediated and the cell-mediated immunity play an important role for achieving clinical immunity [3]. Several studies suggest that successful resolution of malaria infection depends on the ability of the host in inducing adequate levels of pro-inflammatory and regulatory cytokines during key stages of the infection. Thus, the fine-tuning between inflammatory and anti-inflammatory response appears to be a determinant factor in the clinical outcome of the disease [3-5].

Although the mechanisms involved in host immunological response during human malaria are still poorly understood, accumulating data suggest that malaria infection induces pro-inflammatory cytokines that eliminate the parasite or promote the removal of red blood cells infected by the parasite [6]. This response is suppressed in turn by anti-inflammatory cytokines, and the clearance of remaining parasites as well as the prevention of recrudescence or re-infection, are mediated by anti-parasite antibodies [6]. However, depending on factors such as genetic variability of host and parasite, age, number of infections and co-infections, the inflammatory response may be unregulated and, if excessive, it can lead to immunopathology.

Several studies have focused on the profile of plasma cytokines and chemokines in vivax malaria infection, comparing the repertoire of cytokines/chemokines elicited between $P$. vivax and Plasmodium falciparum infection [7-11] and further association with disease severity, determined by clinical symptoms, or immunological profile after treatment with anti-malarial drugs [8-15]. Overall the profile of cytokine/chemokine production is still contradictory due to differences in study population, degree of endemicity in the region, among other factors, requiring further investigations.

The characterization of immune responses elicited during vivax malaria and correlations with the clinical symptoms can reveal important aspects for understanding the pathogenesis of the disease and provide insights for the development of more effective vaccines and even new therapeutic approaches. Because of this, the plasma levels of pro- and anti-inflammatory cytokines and some chemokines were measured in this work during the acute phase of $P$. vivax naturally infected individuals. Samples were also obtained of some patients after anti-malarial drugs treatment to detect possible changes in the host immunological response after treatment and to identify biomarkers of active infection. Finally, analyses of correlation among cytokines/chemokines levels, degree of parasitaemia and number of infections were performed to evaluate whether variations in clinical manifestations are associated with activated or suppressed cytokine/ chemokine networks.

\section{Methods}

\section{Study participants and blood sample collection}

Plasmodium vivax naturally infected individuals with uncomplicated symptomatic malaria ( $P$. vivax group, $\mathrm{n}=75$ ), P.vivax naturally infected individuals after 25 days of treatment with chloroquine and primaquine (P. vivax-treated group, $\mathrm{n}=10$ ) and non-infected subjects with previous episodes of malaria (endemic control group, $\mathrm{n}=10$ ) were recruited at the Centro de Pesquisa em Medicina Tropical (Porto Velho, Rondônia, Brazil). In addition, 15 healthy donors (malaria-naïve control group) with no previous malaria exposure were recruited from a non-endemic area (Belo Horizonte, Minas Gerais, Brazil). The demographic, parasitological and clinical parameters of the subjects are shown in Table 1 . The parasitological demonstration of $P$. vivax infection was performed by well-trained microscopists from the Centro de Pesquisa em Medicina Tropical using thick smears and it was further confirmed by nested polymerase chain reaction (PCR) as previously described [16]. Peripheral venous blood was collected in heparin-containing tubes and centrifuged to obtain plasma. Samples were stored at $-80{ }^{\circ} \mathrm{C}$ until performing the cytokine and chemokine assays.

\section{Cytokine and chemokine plasma levels assays}

Measurements of IL-6, IL-10, IL-17, MCP-1/CCL2, RANTES/CCL5 and IP-10/IP-10/CXCL10 in the plasma samples were conducted using cytometric bead assay (CBA) (BD Biosciences, USA) according to manufacturer's instructions. The data were collected using a FACSCan flow cytometer (BD Biosciences, USA) and the results were analysed in FCAP Array software (Soft Flow). The limit of detection for each assay was: IL- $6=2.4 \mathrm{pg} /$ $\mathrm{mL}, \mathrm{IL}-10=4.5 \mathrm{pg} / \mathrm{mL}, \mathrm{IL}-17=18.9 \mathrm{pg} / \mathrm{mL}, \mathrm{MCP}-1 /$ CCL2 $=2.7 \mathrm{pg} / \mathrm{mL}$, RANTES $/$ CCL $5=1.0 \mathrm{pg} / \mathrm{mL}$ and IP-10/CXCL10 $=2.8 \mathrm{pg} / \mathrm{mL}$. The upper-range limits of detection for the assays were $5000.0 \mathrm{pg} / \mathrm{mL}$ for chemokines (MCP-1/CCL2, RANTES/CCL5 and IP-10/ CXCL10), and $2500.0 \mathrm{pg} / \mathrm{mL}$ for cytokines (IL-6, IL-10 
Table 1 Demographic, parasitological and symptomatological parameters of the study population

\begin{tabular}{|c|c|c|c|c|}
\hline \multirow[t]{2}{*}{ Parameters } & \multicolumn{4}{|c|}{ Value for group } \\
\hline & $\begin{array}{l}\text { Control } \\
(n=15)\end{array}$ & $\begin{array}{l}\text { Endemic } \\
\text { Control } \\
(n=10)\end{array}$ & $\begin{array}{l}\text { P. vivax } \\
(\mathrm{n}=75)\end{array}$ & $\begin{array}{l}P . \text { vivax- } \\
\text { treated } \\
(n=10)\end{array}$ \\
\hline $\begin{array}{l}\text { Age } \\
\text { [median(range)] }\end{array}$ & $27(19-35)$ & $38(21-49)$ & $37(20-80)$ & $42(21-50)$ \\
\hline \multicolumn{5}{|l|}{ Gender [n(\%)] } \\
\hline Male & $10(66.7)$ & $6(60.0)$ & $57(76.0)$ & $6(60.0)$ \\
\hline Female & $5(33.3)$ & $4(40.0)$ & $18(24.0)$ & $4(40.0)$ \\
\hline \multicolumn{5}{|c|}{ Parasitaemia (parasites $\left./ \mathrm{mm}^{3}\right)$, $[\mathrm{n}=(\%)]$} \\
\hline$\leq 500$ & - & - & $34(45.3)$ & - \\
\hline $501-10,000$ & - & - & $26(34.7)$ & - \\
\hline $10,001-100,000$ & - & - & $8(10.7)$ & - \\
\hline $\begin{array}{l}\text { Without informa- } \\
\text { tion }\end{array}$ & - & - & $7(9.3)$ & - \\
\hline \multicolumn{5}{|c|}{ № of previous malaria episodes [n(\%)] } \\
\hline First malaria & - & $3(30.0)$ & $10(13.3)$ & $1(10.0)$ \\
\hline$\leq 5$ & - & $2(20.0)$ & $24(32)$ & $2(20.0)$ \\
\hline$>5$ & - & $5(50.0)$ & $33(44.0)$ & $6(60.0)$ \\
\hline $\begin{array}{l}\text { Without informa- } \\
\text { tion }\end{array}$ & & $0(0.0)$ & $8(10.7)$ & $1(10.0)$ \\
\hline \multicolumn{5}{|l|}{ Symptoms [n(\%)] } \\
\hline Fever & - & - & $66(97.1)$ & - \\
\hline Headache & - & - & $66(97.1)$ & - \\
\hline Myalgia & - & - & $61(89.7)$ & - \\
\hline Chills & - & - & $60(88.2)$ & - \\
\hline Sweating & - & - & $51(75.0)$ & - \\
\hline Arthralgia & - & - & $49(72.1)$ & - \\
\hline Nausea & - & - & $36(52.9)$ & - \\
\hline Vomiting & - & - & $20(29.4)$ & - \\
\hline
\end{tabular}

The parasitaemia and symptoms in the $P$. vivax-treated group refers to acute phase. The $n$ for symptoms were: $P$. vivax group $(n=68)$ and $P$. vivax-treated group $(\mathrm{n}=8)$

and IL-17) detection. Dilution of samples was performed whenever necessary to ensure the obtained values fell within the range of the generated standard curve.

Enzyme-linked immunosorbent assay (ELISA) was performed for the measurement of IL-12p40, IL-27, IFN$\gamma$, TNF and TGF- $\beta$ (R\&D Systems, USA), according to manufacturer's instructions. Biotin-labeled antibodies were used for detection and the assay was revealed with streptavidin-HRP (Amersham Biosciences, USA) using OPD (o-Phenylenediamine dihydrochloride) substrate system (Sigma, USA). The colorimetric reaction was read using an automated ELISA microplate reader (Versamax, Molecular Devices, USA) at $492 \mathrm{~nm}$. The cytokine concentration was calculated from the standard curve using seven-parameter curve fitting software (SOFTmaxPro 5.3, Molecular Devices). The limit of detection for each assay was $156.0 \mathrm{pg} / \mathrm{mL}$ for IL-27, $62.5 \mathrm{pg} / \mathrm{mL}$ for IL-12p40, $31.2 \mathrm{pg} / \mathrm{mL}$ for TGF- $\beta$, and $15.6 \mathrm{pg} / \mathrm{mL}$ for IFN- $\gamma$ and TNF. The upper-range limits of detection for the assays were $10,000.0 \mathrm{pg} / \mathrm{mL}$ for IL-27, $4000.0 \mathrm{pg} / \mathrm{mL}$ for IL-12p40, $2000.0 \mathrm{pg} / \mathrm{mL}$ for TGF- $\beta$, and $1000.0 \mathrm{pg} /$ $\mathrm{mL}$ for IFN- $\gamma$ and TNF. Dilution of samples was performed whenever necessary to ensure the obtained values fell within the range of the generated standard curve.

\section{Statistical analysis}

Statistical analyses were conducted using the Prism software 5.0 for Windows (GraphPad Inc, USA). Initially, Grubb's test was applied to detect possible outliers and the Kolmogorov-Smirnoff test was used to verify the data distribution. Comparisons among groups were performed using Kruskal-Wallis test followed by Dunn's Post-hoc test or Mann-Whitney U test. The paired $t$ test and Wilcoxon test were also applied, according to data distribution. Statistical differences were considered significant when $\mathrm{p}$ values were less or equal to 0.05 . Results were corrected for multiple comparisons as needed.

Correlation networks were generated by the analysis of relationship among cytokine and chemokine plasma level datasets. Initially, pair-wise Spearman correlation coefficients were calculated using a scientific computing library (SciPy) and python programming language. Along with the Spearman rank-order correlation coefficient, the $\mathrm{p}$ value to test for non-correlation was evaluated using $\mathrm{p} \leq 0.05$ as a cut-off. The correlation strength was separated into three ranges: weak $(0.2 \leq \mathrm{r}<0.5)$, moderate $(0.5 \leq \mathrm{r}<0.7)$ and strong $(0.7 \leq \mathrm{r} \leq 1.0)$.

\section{Results}

\section{Plasmodium vivax infection triggers a marked} pro-inflammatory cytokine response

The circulating levels of pro-inflammatory cytokines such as IL-6, IL-17, IL-12p40, and TNF were prominent in $P$. vivax naturally infected individuals when compared to plasma levels observed in other groups (malaria-naïve, endemic and $P$. vivax-treated) (Fig. 1a-d), although significant differences to control groups were observed only for IL-6 and IL-17 ( $p<0.0001$ and $\mathrm{p}=0.0051$, respectively) (Fig. 1a, b). Conversely, the $P$. vivax infection resulted in a significant reduction of IFN- $\gamma$ plasma levels when compared to control groups ( $\mathrm{p}<0.0001)$ (Fig. 1e), with circulating levels of IFN- $\gamma$ detected in only $20 \%$ of the samples from $P$. vivax group.

After treatment, production of IL-6, IL-12p40, TNF and IFN- $\gamma$ were restored to baseline levels ( $\mathrm{p}<0.001$ for IL- 6 and $p<0.05$ for IFN- $\gamma$, only), in contrast to plasma levels of IL-17, which were similar to those presented by infected individuals before treatment. In addition, it was also observed a significant decrease of IL-6 after treatment during a paired analysis (Fig. 1f). 

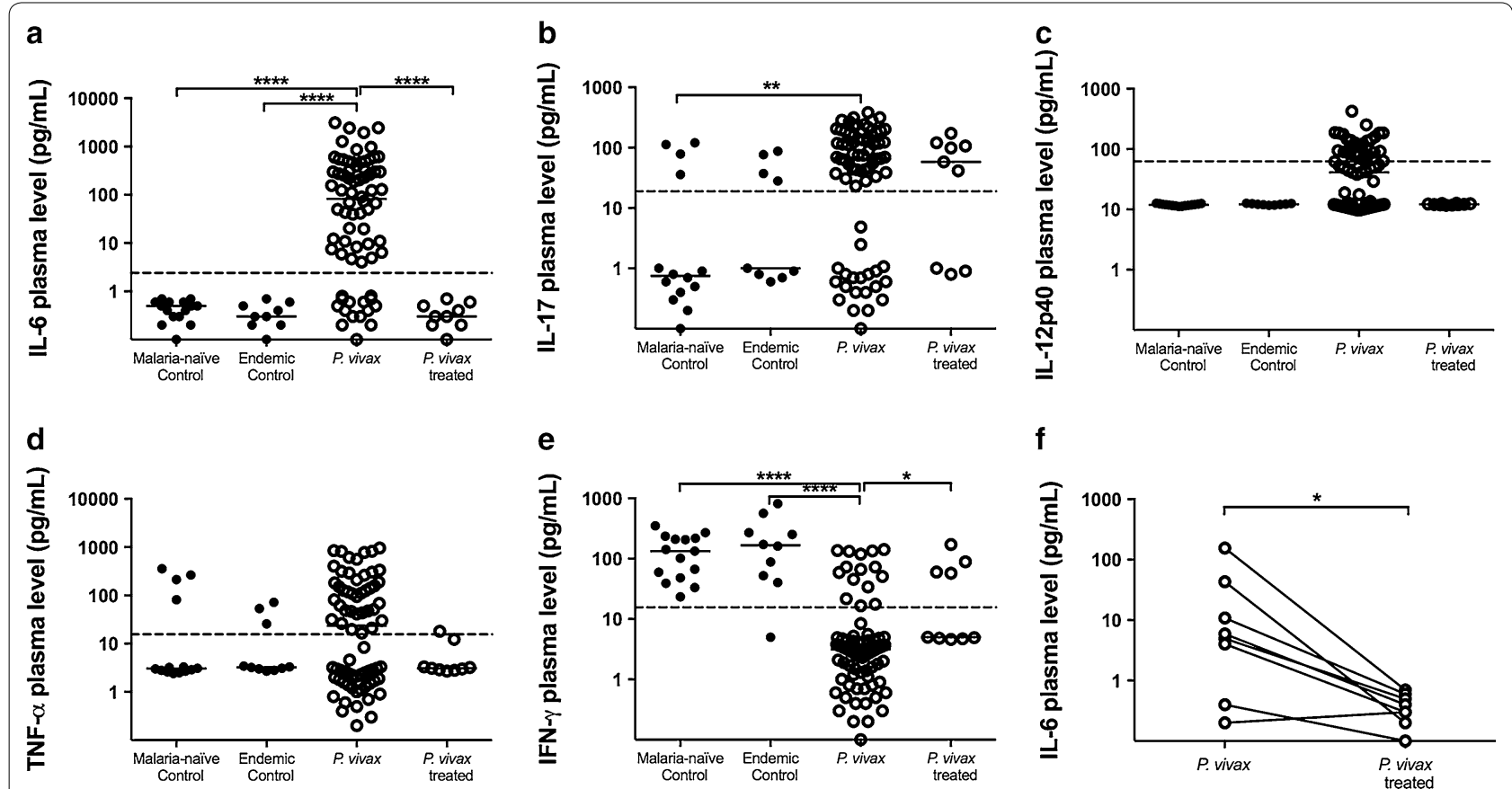

Fig. 1 Inflammatory cytokine plasma levels. Comparative analysis of IL-6 (a), IL-17 (b), IL-12p40 (c), TNF (d) and IFN- - production among malarianaïve control group $(n=15)$, endemic control group $(n=10)$, P. vivax group $(n=75)$ and $P$. vivax-treated group $(n=10)$ were performed using Kruskal-Wallis test followed by Dunn Post-hoc. The dotted lines (-) represent the detection limit of the assay. IL-6 levels in acute-phase and convalescence period from P. vivax-infected patients (e) was compared between P. vivax group $(n=10)$ and $P$. vivax-treated group using Paired $t$-test or Wilcoxon test, according to data distribution. A p value $<0.05$ was considered significant. ${ }^{*} p<0.05,{ }^{* *} p<0.01,{ }^{* * *} p<0.001$, ${ }^{* * * *} p<0.0001$

\section{Higher levels of IL-10 and TGF- $\beta$ were also induced during Plasmodium vivax infection}

Production of IL-10 was only observed in the P. vivax group, being present in $94.4 \%$ of the plasma samples (median $=186.1 \mathrm{pg} / \mathrm{mL})$. After treatment, the IL-10 level returned to basal levels, equivalent to levels observed in control groups $(\mathrm{p}<0.001)$ (Fig. 2a). Similar result was observed for the paired analysis comparing the cytokine levels before and after treatment (Fig. 2b). Moreover, samples from $P$. vivax group presented significant higher levels of TGF- $\beta$ (median $=42.8 \mathrm{pg} /$ $\mathrm{mL}$ ) when compared to malaria-naïve control group $($ median $=10.3 \mathrm{pg} / \mathrm{mL})(\mathrm{p}=0.0353)$ but similar production of this cytokine when compared to endemic control (median $=48.9 \mathrm{pg} / \mathrm{mL}$ ) and $P$. vivax-treated (median $=43.7 \mathrm{pg} / \mathrm{mL}$ ) groups (Fig. $2 \mathrm{c}$ ). No significant differences in the TGF- $\beta$ production was observed for the paired analysis comparing the cytokine level before and after treatment $(\mathrm{p}=0.7344)$.

\section{IL-27 in vivax malaria}

IL-27 is a pleiotropic cytokine that can induce either a pro-inflammatory or immunoregulatory response. The production of IL-27 was reduced in individuals living in endemic areas (endemic control, P. vivax-infected and $P$. vivax-treated individuals). However, significant differences were observed only between the malarianaïve control (median $=1257.0 \mathrm{pg} / \mathrm{mL}$ ) and endemic control $($ median $=105.3 \mathrm{pg} / \mathrm{mL})$ groups $(\mathrm{p}=0.0389$, Fig. 3).

\section{Plasmodium vivax infection induced higher levels of MCP-1/CCL2 and IP-10/CXCL10 and lower levels of RANTES/CCL5}

The plasma levels of MCP-1/CCL2 were increased in $P$. vivax-infected individuals (median $=770.6 \mathrm{pg}$ / $\mathrm{mL}$ ) when compared to malaria-naïve control group (median $=97.3 \mathrm{pg} / \mathrm{mL}$ ) and endemic control group (median $=76.0 \mathrm{pg} / \mathrm{mL}$ ) (Fig. 4a), with further re-establishment to basal levels after treatment $($ median $=104.5 \mathrm{pg} / \mathrm{mL})(\mathrm{p}<0.0001)$. Plasma samples from $P$. vivax-infected donors also presented higher levels of IP-10/CXCL10 in comparison to control groups ( $\mathrm{p}<0.0001$ ) but, similarly to MCP-1/CCL2, the IP-10/ CXCL10 plasma level in treated individuals presented a baseline production that is equivalent to control individuals ( $\mathrm{p}<0.001)$ (Fig. 4b). On the other hand, lower RANTES/CCL5 plasma levels were observed in individuals with $P$. vivax infection when compared to endemic control group $(\mathrm{p}=0.0010)$ (Fig. 4c). 

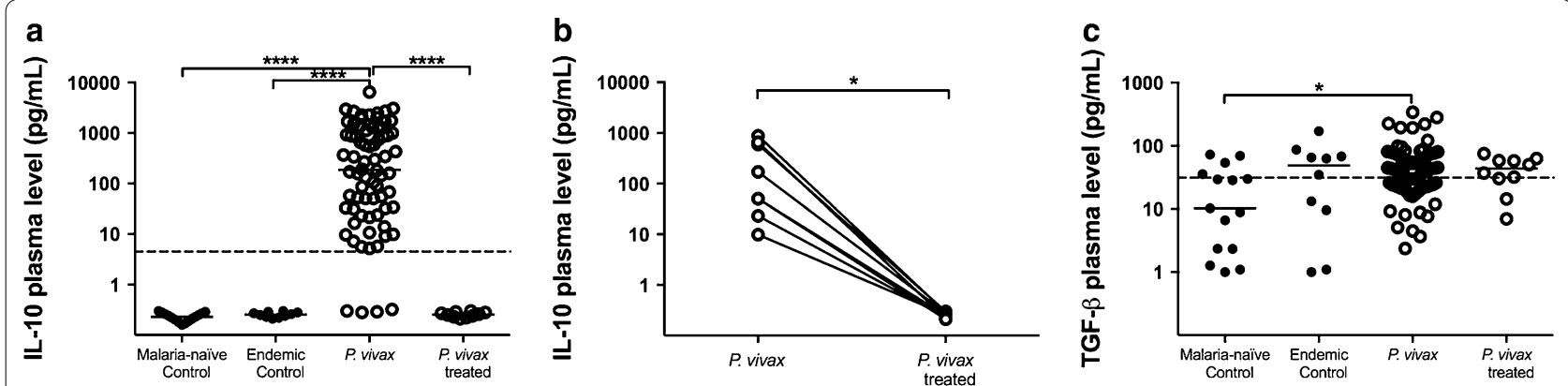

Fig. 2 Regulatory cytokine plasma levels. Comparative analysis of IL-10 (a total production; b paired analysis between infected and treated individuals) and TGF- $\beta$ among malaria-naïve control group $(n=15)$, endemic control group $(n=10)$, P. vivax group ( $n=75)$ and $P$. vivax-treated group ( $n=10$ ) were performed using Kruskal-Wallis test followed by Dunn Post-hoc. A p value $<0.05$ was considered significant. The dotted lines ( - ) represent the detection limit of the assay. Paired analysis was performed between $P$. vivax group $(n=10)$ and $P$. vivax-treated group using Paired t-test or Wilcoxon test, according to data distribution. ${ }^{*} p<0.05,{ }^{* *} p<0.01,{ }^{* *} p<0.001,{ }^{* * *} p<0.0001$

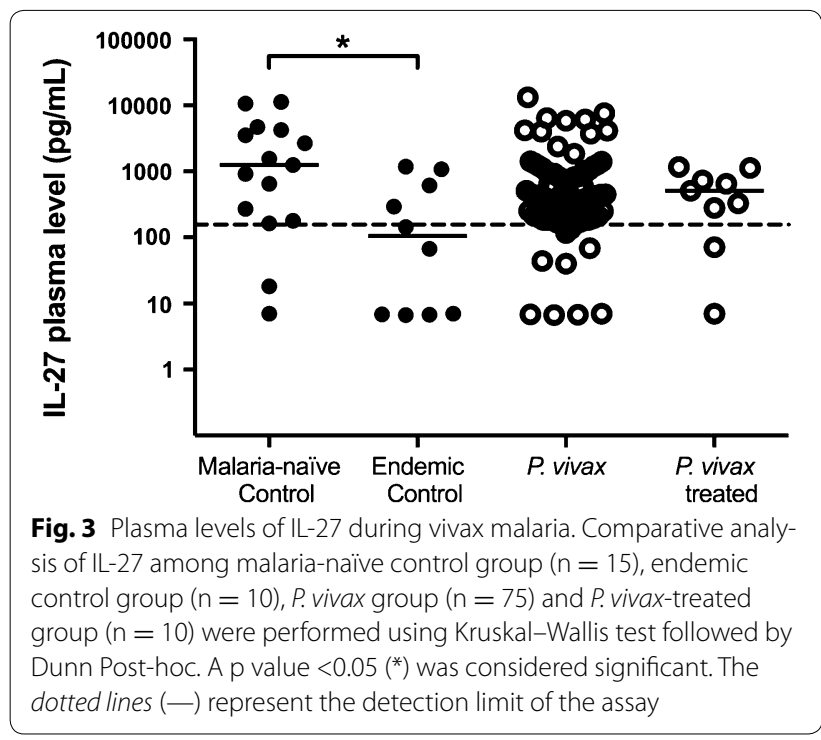

The comparison between plasma levels for both MCP-1/CCL2 and IP-10/CXCL10, before and after treatment (paired analysis), also showed significant differences ( $\mathrm{p}=0.0048$ and $\mathrm{p}=0.0012$, respectively) (Fig. $4 \mathrm{~d}$, e). Despite the absence of significant result between $P$. vivax group and $P$. vivax-treated group due probably to high variability of chemokine levels and differences of sample number, a significant increase was observed in the plasma level of RANTES/CCL5 in the paired t-test after the treatment when following the same individuals (Fig. 4f).

\section{IL-10 plasma levels were associated with parasitaemia and number of previous malaria episodes}

Plasmodium vivax-infected patients were separated into two groups according to parasitaemia: $\leq 500$ parasites/ $\mathrm{cu} \mathrm{mm}$ denominated as low parasitaemia and $>501$ parasites/cu $\mathrm{mm}$ as high parasitaemia (Fig. 5a). Patients with low parasitaemia presented reduced levels of IL-10 (median $=56.2 \mathrm{pg} / \mathrm{mL}$ ) in comparison to patients with high parasitaemia $($ median $=366.1 \mathrm{pg} / \mathrm{mL})(\mathrm{p}<0.0134)$. Furthermore, higher number of previous malaria episodes was associated with higher IL-10 plasma levels ( $<<0.0242)$ (Fig. 5b).

\section{Immune response mediators established a complex network during Plasmodium vivax infection}

During $P$. vivax infection, the triggering of several plasma mediators might result in a complex interaction network, which may render a weak, moderate or strongly correlation among themselves. The network profiles observed in the $P$. vivax group, as well as the sub-groups classified according to the parasite load, are shown in Fig. 6. Interestingly, high parasitaemia network includes practically all correlation observed in the network of all infected patients (Fig. 6a) highlighting the importance of parasite numbers to host immune stimulation. The correlation network between plasma mediators is less connected in low (Fig. 6b) than high parasitaemia (Fig 6c).

Plasmodium vivax-infected patients presented a strong correlation between IL-6 and MCP-1/CCL2 $(r=0.82$, $\mathrm{p}<0.0001)$. Furthermore, moderate correlations were observed between IL- 6 and IL-10 $(r=0.60, \mathrm{p}<0.0001)$ and between IL-12p40 and TNF-a $(r=0.54, \mathrm{p}<0.0001)$. Several weak correlations were observed among other cytokines and chemokines (Fig. 6a). Plasmodium vivax patients were further separated into two sub-groups according to parasitaemia: low ( $\leq 500$ parasites/cu $\mathrm{mm}$ ) and high (>501 parasites/cu mm). Patients with low parasitaemia presented moderate/strong correlations among inflammatory mediators (IL-6/IFN- $\gamma$ and MCP-1/CCL2). On the other hand, only a weak correlation between 

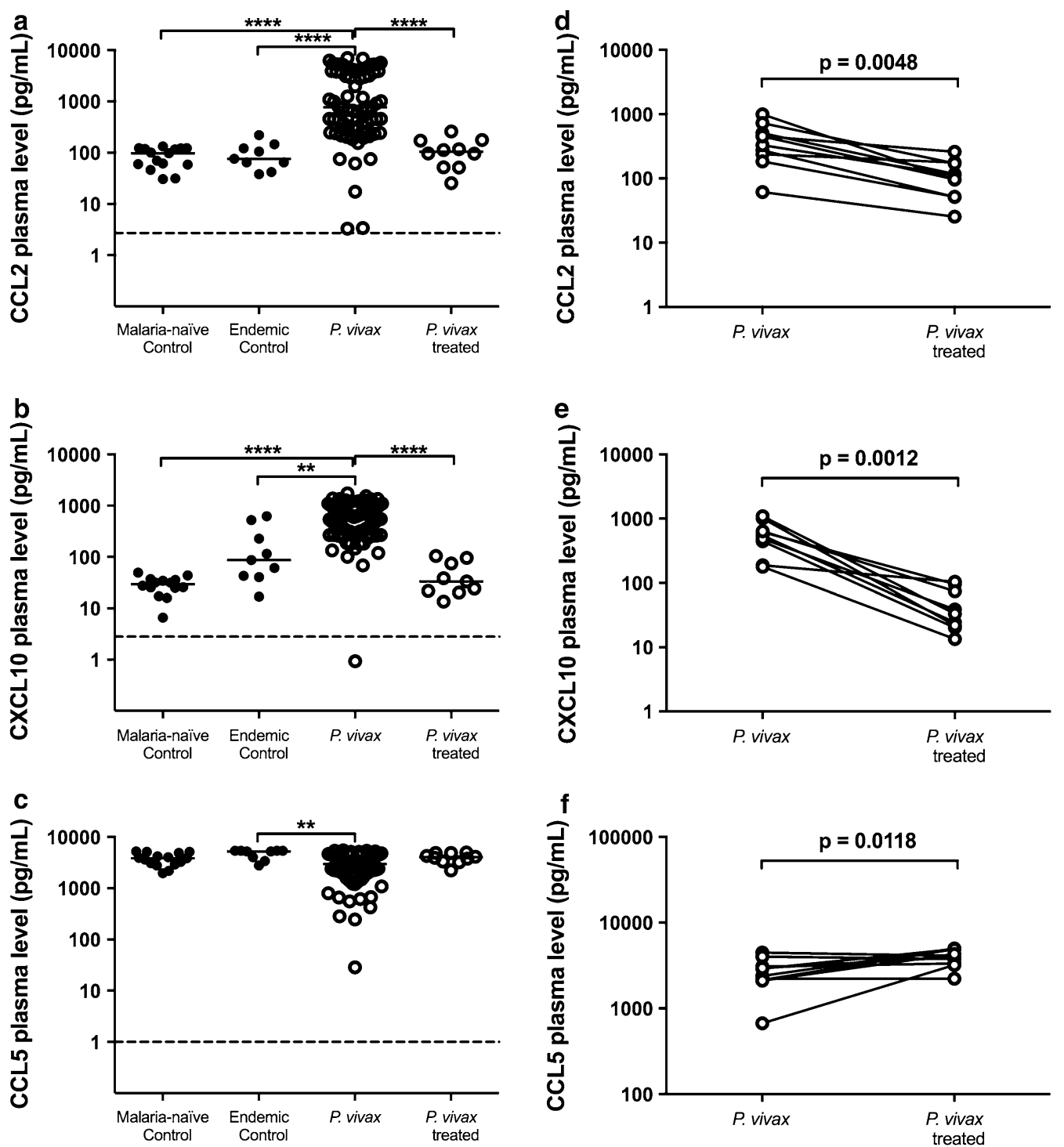

Fig. 4 Chemokine plasma levels. Comparative analysis of MCP-1/CCL2 (a), IP-10/CXCL10 (b), and RANTES/CCL5 (c) production among malaria-naïve control group $(n=15)$, endemic control group $(n=10)$, P. vivax group $(n=75)$ and P. vivax-treated group $(n=10)$ were performed using KruskalWallis test followed by Dunn Post-hoc. The dotted lines (-) represent the detection limit of the assay. Levels of MCP-1/CCL2 (D), IP-10/CXCL10 (e) and RANTES/CCL5 (f) in acute-phase and convalescence period from P. vivax infected patients were determined from P. vivax group $(n=10)$ and P. vivax-treated group using Paired $t$-test or Wilcoxon test, according to data distribution. A $p$ value $<0.05$ was considered significant. ${ }^{*} p<0.05$, ${ }^{* *} p<0.01,{ }^{* * *} p<0.001,{ }^{* * * *} p<0.0001$

IL-6/IL-10 and IL-12p40/TNF was observed in this subpopulation (Fig. 6b). Furthermore, patients with high parasitaemia presented moderate correlations between IL-6 and IP-10/CXCL10 ( $\mathrm{r}=0.56, \mathrm{p}<0.001)$, IL-10 and MCP-1/CCL2 $(\mathrm{r}=0.62, \mathrm{p}<0.001), \mathrm{MCP}-1 / \mathrm{CCL} 2$ and IP-10/CXCL10 ( $\mathrm{r}=0.55, \mathrm{p}<0.001)$ and IL-6/TNF $(\mathrm{r}=0.55, \mathrm{p}<0.001)$ (Fig. 6c).

\section{Discussion}

While some studies have investigated cytokines/ chemokines plasma levels during $P$. vivax infection $[7-15$, 17-19], there is still no agreement regarding the production of cytokines/chemokines and protection. It is well established that a pro-inflammatory response is required for parasite elimination, but an immunomodulatory 

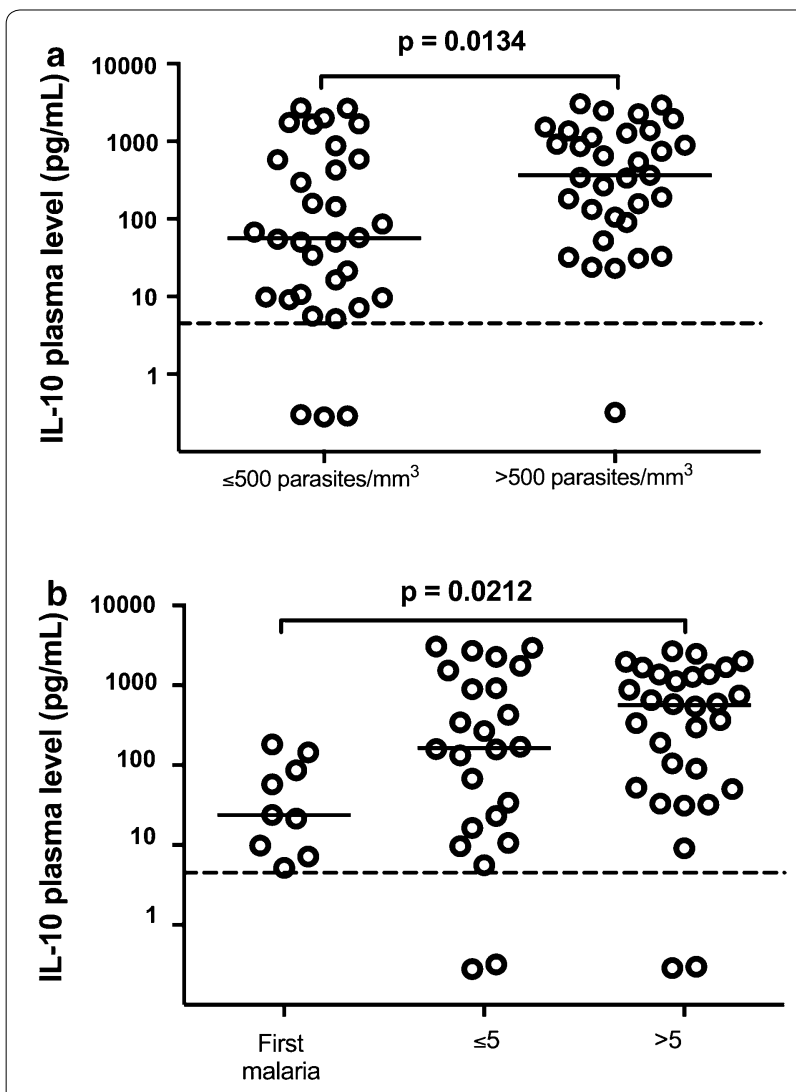

Number of previous malaria episodes

Fig. 5 IL-10 plasma levels and their associations. a Parasitaemia: high vs low number of parasites. Comparison between high ( $>500$ parasites/cu mm, $n=33$ ) and low ( $\leq 500$ parasites/cu mm, $n=32$ ) number of parasites was performed using Mann-Whitney test. $\mathbf{b}$ Number of previous malaria episodes. Comparisons among first malaria $(n=10), \leq 5$ episodes $(n=24)$ and $>5$ episodes $(n=32)$ were performed using Kruskal-Wallis test followed by Dunn Post-hoc A $p$ value $<0.05$ was considered significant. ${ }^{*} p<0.05$. The dotted lines (-) represent the detection limit of the assay

response is also needed to prevent immunopathology [6]. In the present study, it was shown that $P$. vivax infection induced increased levels of IL-6 and, after treatment, the plasma levels were restored. These findings were in agreement with previous studies during acute phase [11, $15,17,18]$ although the controversial data in the literature with reduction $[10,14]$ or increase [11] of IL- 6 after the treatment, which might be attributed to convalescence period range ( $7-45$ days) in the different studies [8, $10,11,13,14]$.

Higher levels of some pro-inflammatory mediators such as IL-6, MCP-1/CCL2 and IP-10/CXCL10 were observed in $P$. vivax-infected patients, which were re-established after anti-malarial treatment, suggesting that the parasite infection triggered an inflammatory response. Concomitantly, it seems to be a consensus that $P$. vivax infection induces higher levels of IL-10 during acute phase of vivax malaria $[7,8,10-13,15,17-19]$ and that the cytokine levels were restored to baseline after treatment $[8,10$, $11,14]$. Indeed, a previous study demonstrated that $\mathrm{CD} 4{ }^{+} \mathrm{CD} 25^{+} \mathrm{T}$ cells producing IL-10 play a significant role during Plasmodium infection, possibly controlling the pro-inflammatory cytokines IFN- $\gamma$ and TNF [6]. Of note, previous studies also demonstrated the increased number of circulating Treg cells $\left(\mathrm{CD} 4{ }^{+} \mathrm{CD} 25^{+}\right.$Foxp $\left.3^{+}\right)$ during $P$. vivax infection [20, 21]. Regarding the association between IL-10 plasma levels with parasitaemia and number of previous malaria episodes, in the present study, it was observed higher IL-10 plasma levels in patients with high parasitaemia ( $>501$ parasites/cu $\mathrm{mm}$ ) and with more than five previous malaria episodes. Similar result was observed for the association with parasitaemia, but not with number of previous malaria episodes [18]. The significance of IL-6 and IL-10 has been highlighted during vivax malaria. High levels of these cytokines were observed in uncomplicated cases [18, 22], while low IL-6 levels were observed in patients with complicated malaria [22]. In addition, a positive correlation between IL- 6 and IL-10 has also been observed in $P$. vivax infection $[10,18,22]$. Likewise, in the present study correlation analysis between IL-6 and IL-10 demonstrated a positive correlation $(\mathrm{r}=0.60)$ in $P$. vivax group, suggesting the acquisition of a more immunomodulatory profile. The association between IL-10 plasma levels and high parasitaemia could reflect a self-regulation mechanism to protect the excessive inflammatory response because of high antigen stimulation.

During acute episode of vivax malaria, several studies have reported high plasma levels of IFN- $\gamma[7,10,12$, $15,19]$. In the present study, IFN- $\gamma$ plasma levels were only observed in $20 \%$ of $P$. vivax patient samples. On the other hand, the majority of patients of this group presented higher production of IL-17. However, reports on IL-17 production in the human malarial infection are poorly described $[9,11]$ and further investigations are required. It is important to highlight that IL-17 may be produced by macrophages, dendritic cells, NK, NKT, $\gamma \mathrm{dT}$ cells, $\mathrm{CD}^{+}$and Th17 $[23,24]$ and the source of this cytokine during $P$. vivax infection needs more investigation. A previous study showed that $\mathrm{CD}^{+}{ }^{+} \mathrm{T}$ cells producing IL-17 were increased during vivax malaria [25]. The higher plasma levels of TGF- $\beta$ and IL- 6 in the acute phase could suggest the induction of IL-17, since naive $\mathrm{CD} 4^{+} \mathrm{T}$ cells require stimulation by IL- 6 and TGF- $\beta$, and possibly IL-1b, to differentiate in Th17 and to secrete IL-17 [23, 26, 27]. Th17 cells induced by IL- 6 and TGF- $\beta$ also produce IL-10, presenting a regulatory function [28]. It is important to highlight that the low levels of IFN- $\gamma$ observed in P. vivax group could contribute to high levels 


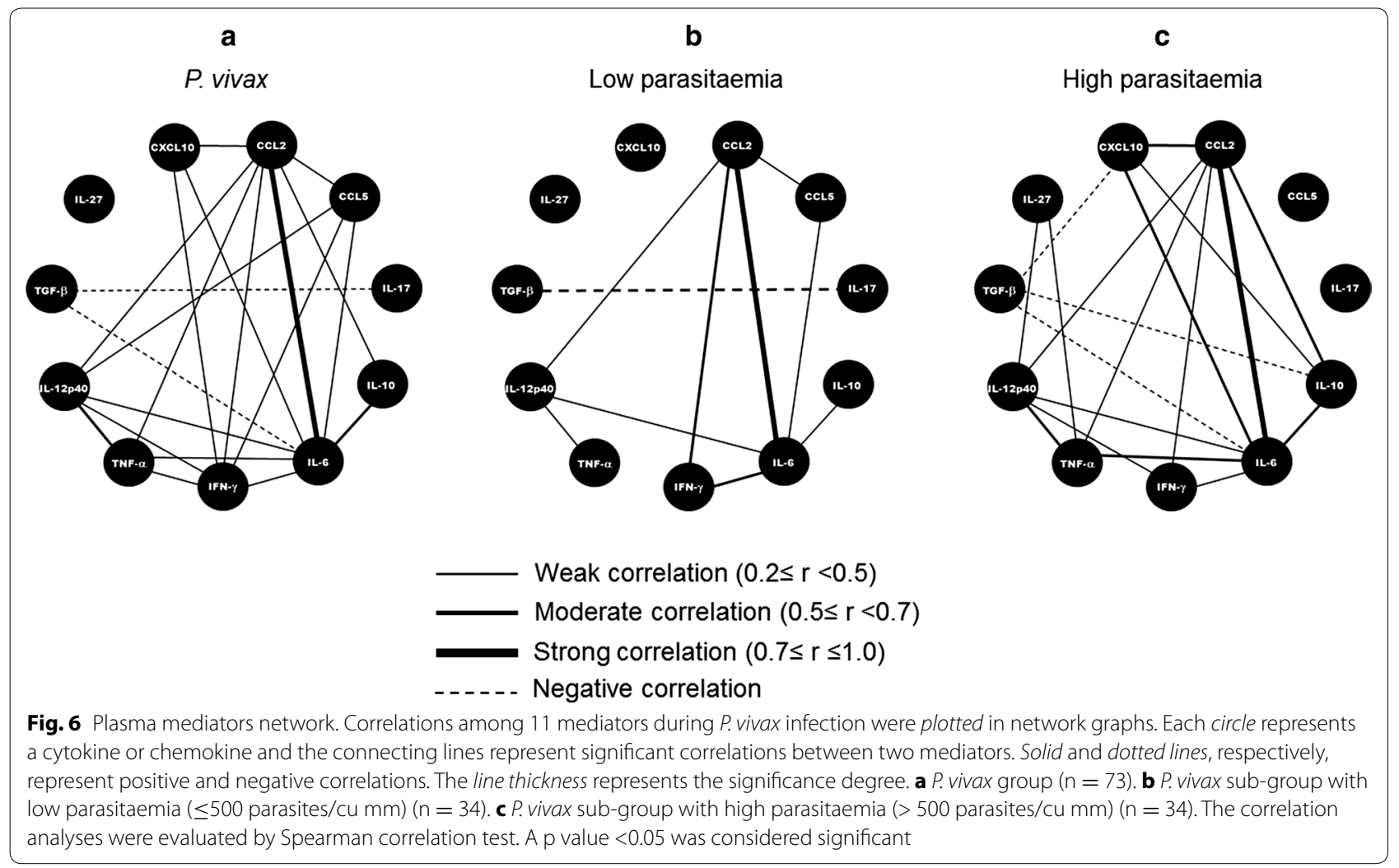

of IL-17 once IFN- $\gamma$ negatively regulates the generation of IL-17-producing cells [29].

Despite the high TGF- $\beta$ plasma levels observed during $P$. vivax infection, there was no consensus about these findings $[8,10,15]$. TGF- $\beta$ is a potent inductor of Treg cells [30], which may have contributed to the high IL-10 plasma levels observed. Although high IL-17 plasma levels were observed in $P$. vivax group, only a weak negative correlation was detected between IL-17 and TGF- $\beta$ by the network analysis approach. This connection was also found in low parasite load sub-group. Therefore, the IL-17 and TGF- $\beta$ roles during $P$. vivax infection require additional investigations. Regarding IL-12p40 and TNF, no significant results were observed in the present study. However, differences in literature findings occur for both cytokines in acute phase [7, 8, 10-13, 15, 17-19] and convalescence period $[8,10,11,13,14]$.

IL-27 is a pleiotropic cytokine with both pro- and antiinflammatory actions. This cytokine is a potent inhibitor of Th17 cell development and of IL-17 induction [31, 32]. In the present study, low IL-27 levels were observed in endemic control group compared to malaria-naïve control group. However, no difference was observed regarding to IL-17 between the control groups. In children infected with $P$. falciparum, IL-27 plasma levels were decreased during uncomplicated malaria in comparison to endemic control group. This reduction was more accentuated in severe cases [33]. Thus, the IL-27 role in vivax malaria needs further investigation.

MCP-1/CCL2, known as Monocyte Chemoattractant Protein-1, is a powerful attractant of monocytes, $\mathrm{T}$ cells and dendritic cells to inflammatory sites. This chemokine is produced by several cell types, such as epithelial, endothelial, smooth muscle, fibroblasts, astrocytes, monocytes, and microglial cells, and can be induced by TNF, IL-1 and endotoxins [34]. In the present study, high plasma levels of MCP-1/CCL2 were observed during $P$. vivax infection. Similar result was previous described during $P$. vivax infection [7]. The pro-inflammatory IL- 6 cytokine induced the mRNA expression and MCP-1 secretion by peripheral blood mononuclear cells [35]. During blood stage of P. vivax infection both IL-6 and MCP-1/CCL2 were significantly elevated. The network analysis revealed a strong correlation between IL-6 and MCP-1/CCL2, regardless of parasite load, suggesting that these mediators were induced by the infection itself. These findings support the hypothesis that IL-6 and MCP-1/CCL2 pathway plays a central role in response to $P$. vivax infection. Interestingly, the network analyses for the high and low parasitaemia groups have shown that individuals with high parasitaemia exhibit moderate/strong correlation between IL-6/IL-10 and MCP-1/ CCL2. However, patients with low parasitaemia exhibited weak correlation between IL- 6 and IL-10, losing the 
interaction between IL-10 andMCP-1/CCL2. These data associated with the positive correlation between IL-6/ IFN- $\gamma$ reinforce the significance of the IL-6/MCP-1/ IFN- $\gamma$ axis in controlling parasitaemia, which could contribute to the lower parasite load observed.

IP-10/CXCL10 (IFN-inducible protein 10) is another chemokine that is induced by IFN- $\gamma$ [36] as well as by IL-17 [37] in different cell types. This chemokine is involved in inflammatory processes, being capable of attracting macrophages, dendritic cells, NK cells and activated $\mathrm{CD}_{4}^{+}$and $\mathrm{CD} 8^{+} \mathrm{T}$ cells towards inflamed tissues [36]. In the present study, P. vivax infected patients presented elevated IP-10/CXCL10 plasma levels, but only weak positive correlation with IFN- $\gamma$. No correlation was observed between IP-10/CXCL10 and IL-17, although both mediators were elevated during $P$. vivax infection. However, when patients were separated according to the parasite load, this connection was lost, and moderate correlation was established between IP-10/CXCL10 and IL-6, only in patients with high parasitaemia. In $P$. falciparum infection, IP-10/CXCL10 has been identified as biomarker (in serum and cerebrospinal fluid) associated with elevated risk of fatal cerebral malaria [38]. On the other hand, higher IP-10/CXCL10 plasma levels, as well as IFN- $\gamma$ and IL-10, were observed in vivax malaria patients with mild anaemia in comparison to no anaemia [12].

RANTES/CCL5, known as Regulated upon Activation Normal T cell Expressed and Secreted, is an inflammatory chemokine attractant of $\mathrm{T}$ cells, basophils, eosinophils, and dendritic cells to inflammatory site [39]. RANTES/CCL5 is produced predominantly by CD8 ${ }^{+}$ $\mathrm{T}$ cells, epithelial cells, fibroblasts, and platelets [40]. In children infected by falciparum malaria low mRNA and RANTES protein levels were associated with severe malaria [41]. Lower RANTES levels were also found in children with cerebral malaria and a strong positive correlation was verified between RANTES levels and platelets count [42]. In the present study, vivax malaria patients have shown significant low RANTES/CCL5 levels, but just weakly associated with IL-6, IL-12p40, IFN- $\gamma$ or MCP-1/CCL2. The lower levels of RANTES/CCL5 could be explained by the $\mathrm{CD}^{+} \mathrm{T}$ cells reduction [43$45]$ and thrombocytopaenia [8, 10, 11, 44-46] observed during vivax malaria. A study carried out with children infected with $P$. falciparum observed an association between thrombocytopaenia and lower RANTES plasma levels [47].

\section{Conclusion}

Taken together, the multiple analyses performed in the present study allowed the identification of an immunological signature from plasma mediators associated with
P. vivax acute infection. IL-6, MCP-1/CCL2 and IL-10 could be recognized as biomarkers of acute phase of $P$. vivax infection. These results provide new insights into the complex relationship among mediators that are triggered during $P$. vivax clinical malaria.

\section{Authors' contributions}

NSHS, DBP, FSHS, TAOM, MSC, RTF, and LLB conceived and designed the experiments; NSHS and DBP performed the experiments; NSHS, DBP, FSHS, MSC, TAOM, RTF and LLB analysed the data; NSHS, DBP, FSHS, TAOM, MSC, GMZ, DCB, RTF and LLB contributed reagents/materials/analysis tools; NSHS, DBP, FSHS, TAOM, MSC, DCB, RTF, and LLB wrote the paper; DBP and MST assisted with patient care and case identification. All authors read and approved the final manuscript.

\section{Author details \\ 1 Departamento de Parasitologia, Instituto de Ciências Biológicas, Universi- dade Federal de Minas Gerais, Belo Horizonte, Minas Gerais, Brazil. ${ }^{2}$ Centro de Pesquisa em Medicina Tropical, Porto, Velho, Rondônia, Brazil. ${ }^{3}$ Departamento de Ciência da Computação, Universidade Federal de São João del-Rei, São João del-Rei, Minas Gerais, Brazil. ${ }^{4}$ Departamento de Bioquímica e Biologia Molecular, Universidade Federal de Viçosa, Viçosa, Minas Gerais, Brazil. ${ }^{5}$ Insti- tuto de Pesquisa Clínica Evandro Chagas, Fundação Oswaldo Cruz, Rio de Janeiro, Rio de Janeiro, Brazil.}

\section{Acknowledgements}

NSH-S was supported by a doctoral degree fellowship from CNPq/Brazil. RF and DB are supported by Brazilian National Research Council (CNPq) fellowships.

\section{Competing interests}

The authors declare that they have no competing interests.

\section{Availability of data and materials}

All data and materials are available upon request.

\section{Ethics approval and consent to participate}

The present study was approved by the Ethics Committee of the Centro de Pesquisa em Medicina Tropical (CAAEs: 0008.0.046.000-11, 0449.0.203.000-09) and the Ethics Committee of the Universidade Federal de Minas Gerais (CAAE: 27466214.0.0000.5149). Written informed consent was obtained from each participant.

\section{Funding}

This work was financially supported by Conselho Nacional de Desenvolvimento Científico e Tecnológico/CNPq (Grant \#478379/2013-7), FAPEMIG (Grant \# APQ-00814-15) and Pró-Reitoria de Pesquisa of Universidade Federal de Minas Gerais.

Received: 8 November 2016 Accepted: 5 January 2017

Published online: 24 January 2017

\section{References}

1. WHO. World malaria report 2014. Geneva: World Health Organization; 2014. http://www.who.int/malaria/publications/world_malaria_ report_2014/en/. Accessed 15 Nov 2014.

2. Struik SS, Riley EM. Does malaria suffer from lack of memory? Immunol Rev. 2004:201:268-90.

3. Artavanis-Tsakonas K, Tongren JE, Riley EM. The war between the malaria parasite and the immune system: immunity, immunoregulation and immunonopathology. Clin Exp Immunol. 2003;133:145-52.

4. Finney OC, Riley EM, Walther M. Regulatory T cells in malaria-friend or foe? Trends Immunol. 2010;31:63-70.

5. Hansen DS, Schofield L. Natural regulatory T cells in malaria: host or parasite allies? PLoS Pathog. 2010;6:e1000771.

6. Riley EM. Regulating immunity to malaria. Parasite Immunol. 2006:28:35-49. 
7. Fernandes AAM, Carvalho LJM, Zanini GM, Ventura AMRS, Souza JM, Cotias PM, et al. Similar cytokine responses and degrees of anemia in patients with Plasmodium falciparum and Plasmodium vivax infections in the Brazilian Amazon region. Clin Vaccine Imunol. 2008;15:650-8.

8. Gonçalves RM, Salmazi KC, Santos BAN, Bastos MS, Rocha SC, Boscardin $\mathrm{SB}$, et al. $\mathrm{CD} 4^{+} \mathrm{CD} 25^{+}$Foxp $3^{+}$regulatory T cells, dendritic cells, and circulating cytokines in uncomplicated malaria: do different parasite species elicit similar host responses? Infect Immun. 2010;78:4763-72.

9. Cox-Singh J, Singh B, Daneshvar C, Planche T, Parker-Williams J, Krishna S. Anti-inflammatory cytokines predominate in acute human Plasmodium knowlesi infections. PLoS ONE. 2011;6:e20541.

10. Gonçalves RM, Scopel KKG, Bastos MS, Ferreira MU. Cytokine balance in human malaria: does Plasmodium vivax elicit more inflammatory responses than Plasmodium falciparum? PLoS ONE. 2012;7:e44394.

11. Rodrigues-da-Silva RN, Lima-Junior JC, Fonseca BPF, Antas PRZ, Baldez A, Storer $\mathrm{FL}$, et al. Alterations in cytokines and haematological parameters during the acute and convalescent phases of Plasmodium falciparum and Plasmodium vivax infections. Mem Inst Oswaldo Cruz. 2014;109:154-62.

12. Jain V, Singh PP, Silawat N, Patel R, Saxena A, Bharti PK, et al. A preliminary study on pro- and anti-inflammatory cytokine profiles in Plasmodium vivax malaria patients from central zone of India. Acta Trop. 2010;113:263-8.

13. Andrade BB, Reis-Filho A, Souza-Neto SM, Clarêncio J, Camargo LMA, Barral $A$, et al. Severe Plasmodium vivax malaria exhibits marked inflammatory imbalance. Malar J. 2010;9:13.

14. Leoratti FMS, Trevelin SC, Cunha FQ, Rocha BC, Costa PAC, Gravina HD, et al. Neutrophil paralysis in Plasmodium vivax malaria. PLoS Negl Trop Dis. 2012;6:e1710.

15. Mendonça VRR, Queiroz ATL, Lopes FM, Andrade BB, Barral-Neto M. Networking the host immune response in Plasmodium vivax malaria. Malar J. 2013;12:69.

16. Snounou G, Viriyakosol S, Zhu XP, Jarra W, Pinheiro L, Rosário VE, et al. High sensitivity of detection of human malaria parasites by the use of nested polymerase chain reaction. Mol Biochem Parasitol. 1993;61:315-20.

17. Zeyrek FY, Kurcer MA, Zeyrek D, Simsek Z. Parasite density and serum cytokine levels in Plasmodium vivax malaria in Turkey. Parasite Immunol. 2006:28:201-7.

18. Costa AG, Antonelli LRV, Costa PAC, Pimentel JPD, Garcia NP, Tarragô AM, et al. The robust and modulated biomarker network elicited by the Plasmodium vivax infection is mainly mediated by the IL-6/IL-10 axis and is associated with the parasite load. J Immunol Res. 2014;2014:318250.

19. Medina TS, Costa SPT, Oliveira MD, Ventura AM, Souza JM, Gomes TF, et al. Increased interleukin-10 and interferon- $\gamma$ levels in Plasmodium vivax malaria suggest a reciprocal regulation which is not altered by $\mathrm{IL}-10$ gene promoter polymorphism. Malar J. 2011;10:264.

20. Jangpatarapongsa K, Chootong P, Sattabongkot J, Chotivanich K, Sirichaisinthop J, Tungpradabkul S, et al. Plasmodium vivax parasites alter the balance of myeloid and plasmacytoid dendritic cells and the induction of regulatory T cells. Eur J Immunol. 2008;38:2697-705.

21. Bueno LL, Morais CG, Araújo FF, Gomes JAS, Corrêa-Oliveira R, Soares IS, et al. Plasmodium vivax: induction of $\mathrm{CD}^{+} \mathrm{CD}_{2} 5^{+} \mathrm{FoxP} 3^{+}$regulatory $T$ cells during infection are directly associated with level of circulating parasites. PLoS ONE. 2010;5:e9623.

22. Raza A, Ghanchi NK, Raheem A, Nizami S, Beg MA. Tumor necrosis factor-a, interleukin-10, intercellular and vascular adhesion molecules are possible biomarkers of disease severity in complicated Plasmodium vivax isolates from Pakistan. PLoS ONE. 2013;8:e81363.

23. Onishi RM, Gaffen SL. Interleukin-17 and its target genes: mechanisms of interleukin-17 function in disease. Immunology. 2010;129:311-21.

24. Xu S, Cao X. Interleukin-17 and its expanding biological functions. Cell Mol Immunol. 2010;7:164-74

25. Bueno LL, Morais CG, Lacerda MV, Fujiwara RT, Braga EM. Interleukin-17 producing T helper cells are increased during natural Plasmodium vivax infection. Acta Trop. 2012;123:53-7.

26. Romagnani S. Human Th17 cells. Arthritis Res Ther. 2008:10:206
27. Awasthi A, Kuchroo VK. Th17 cells: from precursors to players in inflammation and infection. Int Immunol. 2009;21:489-98.

28. McGeachy MJ, Bak-Jensen KS, Chen Y, Tato CM, Blumenschein W, McClanahan T, Cua DJ. TGF-beta and IL-6 drive the production of IL-17 and IL-10 by $T$ cells and restrain $T_{H^{-1}} 17$ cell-mediates pathology. Nat Immunol. 2007;8:1390-7.

29. Park H, Li Z, Yang XO, Chang SH, Nurjeva R, Wang YH, et al. A distinct lineage of CD4 T cells regulates tissue inflammation by producing interleukin 17. Nat Immunol. 2005;6:1133-41.

30. Betelli E, Carrier Y, Gao W, Korn T, Strom TB, Oukka M, et al. Reciprocal developmental pathways for the generation of pathogenic effector $\mathrm{TH} 17$ and regulatory T cells. Nature. 2006:441:235-8.

31. Stumhofer SJ, Hunter CA. Advances in understanding the anti-inflammatory properties of IL-27. Immunol Lett. 2008;117:123-30.

32. Murugaiyan G, Mittal A, Lopez-Diego R, Maier LM, Anderson DE, Weiner $\mathrm{HL}$. IL-27 is a key regulator of IL-10 and IL-17 production by human CD4 ${ }^{+}$ T Cells. J Immunol. 2009;183:2435-43.

33. Ayimba E, Hegewald J, Ségbéna AY, Gantin RG, Lechner CJ, Agossou $A$, et al. Proinflammatory and regulatory cytokines and chemokines in infants with uncomplicated and severe Plasmodium falciparum malaria. Clin Exp Immunol. 2011;166:218-26.

34. Yadav A, Saini V, Arora S. MCP-1: chemoattractant with a role beyond immunity: a review. Clin Chim Acta. 2010;411:1570-9.

35. Biswas P, Delfanti F, Bernasconi S, Mengozzi M, Cota M, Polentarutti N, et al. Interleukin-6 induces monocyte chemotactic protein-1 in peripheral blood mononuclear cells in the U937 cell line. Blood. 1998;91:258-65.

36. Liu M, Guo S, Hibbert JM, Jain V, Singh N, Wilson NO, Stiles JK. IP-10/ CXCL10/P-10 in infectious disease pathogenesis and potential therapeutic implications. Cytokine Growth Factor Rev. 2011;22:121-30.

37. Weaver CT, Hatton RD, Mangan PR, Harrington LE. IL-17 family cytokines and the expanding diversity of effector T cell lineages. Annu Rev Immunol. 2007; 25:821-52.

38. Armah HB, Wilson NO, Sarfo BY, Powell MD, Bond VC, Anderson W, et al. Cerebrospinal fluid and serum biomarkers of cerebral malaria mortality in Ghanaian children. Malar J. 2007;6:147.

39. Arango Duque G, Descoteaux A. Macrophage cytokines: involvement in immunity and infectious disease. Front Immunol. 2014;5:491.

40. Appay V, Rowland-Jones SL. RANTES: a versatile and controversial chemokine. Trends Immunol. 2001;22:83-7.

41. Ochiel DO, Awandare GA, Keller CC, Hittner JB, Kremsner PG, Weinberg JB, et al. Differential regulation of beta-chemokines in children with Plasmodium falciparum malaria. Infect Immun. 2005;73:4190-7.

42. John CC, Opika-Opoka R, Byarugaba J, Idro R, Boivin MJ. Low levels of RANTES are associated with mortality in children with cerebral malaria. J Infect Dis. 2006;194:837-45.

43. Kassa D, Petros B, Mesele T, Hailu E, Wolday D. Characterization of peripheral blood lymphocyte subsets in patients with acute Plasmodium falciparum and P. vivax malaria infections at Wonji Sugar Estate, Ethiopia. Clin Vaccine Immunol. 2006;13:376-9.

44. Borges QI, Fontes CJF, Damazo AS. Analysis of lymphocytes in patients with Plasmodium vivax malaria and its relation to the annexin-A1 and IL-10. Malar J. 2013;12:455

45. Hojo-Souza NS, Pereira DB, Passos LS, Gazzinelli-Guimarães PH, Cardoso $\mathrm{MS}$, Tada MS, et al. Phenotypic profiling of $\mathrm{CD} 8^{+} \mathrm{T}$ cells during Plasmodium vivax blood-stage infection. BMC Infect Dis. 2015;15:35.

46. Hojo-Souza NS, Pereira DB, Mendes TA, Passos LS, Gazzinelli-Guimarães AC, Gazzinelli-Guimarães $\mathrm{PH}$, et al. CD4 ${ }^{+} \mathrm{T}$ cells apoptosis in Plasmodium vivax infection is mediated by activation of both intrinsic and extrinsic pathways. Malar J. 2015;14:5.

47. Were T, Hittner JB, Ouma C, Otieno RO, Orago AS, Ong'echa JM, et al. Suppression of RANTES in children with Plasmodium falciparum malaria. Haematologica. 2006;91:1396-9. 